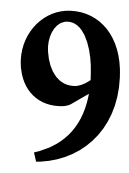

\title{
LA COLABORACIÓN INTERINSTITUCIONAL COMO RESPUESTA AL DESAFÍO DE LA INCLUSIÓN SOCIOEDUCATIVA. ANÁLISIS DE 4 EXPERIENCIAS DE TRABAJO EN RED A NIVEL LOCAL EN CATALUÑA
}

\author{
(INTER-INSTITUTIONAL COLLABORATION IN RESPONSE TO THE \\ CHALLENGE OF SOCIO-EDUCATIONAL INCLUSION. ANALYSIS OF 4 \\ TERRITORIAL NETWORKING EXPERIENCES IN CATALONIA)
}

Mireia Civís Zaragoza y Jordi Longás Mayayo Universitat Ramon Llull

DOI: 10.5944/educXX1.18.1.12318

Cómo referenciar este artículo/How to reference this article:

Civís Zaragoza, M.; Longás Mayayo, J. (2015). La colaboración interinstitucional como respuesta al desafío de la inclusión socioeducativa. Análisis de 4 experiencias de trabajo en red a nivel local en Cataluña. Educación XX1, 18(1), 213-236. doi: 10.5944/educXX1.18.1.12318

Civís Zaragoza, M.; Longás Mayayo, J. (2015). Inter-institutional collaboration in response to the challenge of socio-educational inclusion. Analysis of 4 territorial networking experiences in Catalonia. Educación XX1, 18(1), 213-236. doi: 10.5944/educXX1.18.1.12318

\section{RESUMEN}

El artículo reflexiona en torno a la emergencia del trabajo socioeducativo en red, articulado a partir de la colaboración entre instituciones sociales y educativas. Se presenta el concepto de red socioeducativa y el sentido que tiene plantear esta nueva forma de organizar la acción educativa y social que sitúa a la escuela en el núcleo de su entramado. Así mismo, se discuten los principios propios de este modelo emergente de acción socioeducativa y los retos de su gestión. La proximidad, transversalidad, horizontalidad, corresponsabilidad, colaboración, proactividad y proyección articulan y organizan la acción socioeducativa, la cual requiere liderazgos capaces de desarrollar la red y lograr los objetivos planteados.

Así mismo, y mediante el contraste entre la aproximación teórica y 4 reconocidas experiencias de trabajo socioeducativo en red a nivel local en Cataluña, se destacan los factores comunes que se observan y se valora el interés y viabilidad del modelo presentado. Se trata de redes para la mejora de la inclusión social y educativa de adolescentes y jóvenes, con el énfasis puesto en 
el éxito escolar y los procesos de transición escuela trabajo (TET). Se analiza su evolución, finalidad, organización y logros.

El artículo concluye acerca de la importancia de un modelo de trabajo compartido, redistribuido y democratizado para atender los desafíos comunes, evitando los modelos donde la escuela centraliza toda la acción o bien los modelos donde se excluye a la escuela para dar protagonismo a otros agentes.

\section{PALABRAS CLAVE}

Educación comunitaria, relación escuela-comunidad, desarrollo comunitario, éxito escolar, redes educativas locales.

\section{ABSTRACT}

This article reflects on the emergence of socio-educational networking, set up from the collaboration among social and educational institutions. Firstly, the concept of network and the sense of proposing this new way of organizing educational and social action with the school at the core are presented. Then, the principles of this emergent model of socio-educational action are discussed, with network management also being tackled. The principles of proximity, transversality, horizontality, co-responsibility, collaboration, proactivity and projection express and organize the educational action, which requires a leadership capable of developing the network and achieving the objectives.

Also, by contrasting the theoretical approach and 4 recognized socio-educational networking experiences in Catalonia, common factors are highlighted and the interest and feasibility of the model presented is appreciated. They are networks that focus on improving social and educational inclusion of adolescents and young people with stress placed on school success and the school to work transition. Its evolution, purpose, organization and performance are analyzed.

The paper reaches conclusions about the importance of shared, redistributed and democratized work in order to meet common challenges, avoiding models where the school centralizes all the action or models where the school is excluded to give prominence to other agents.

\section{KEY WORDS}

Community education, school-community relationship, community development, school success, local educational networks. 


\section{INTRODUCCIÓN}

Aunque parece asumido que educación y escolarización no son lo mismo, el análisis de la política y práctica educativa no muestra de forma tan evidente que las instituciones, los profesionales y la sociedad así lo comprendan. La escuela todavía sigue colapsada por un exceso de demandas sociales y por la atribución de responsabilidades que la desbordan, en una muestra de la confusión que existe sobre quién debe hacer qué en el terreno educativo. Con frecuencia, esta sobrecarga educativa de la escuela indica el desconcierto y/o la escasa responsabilización de muchos agentes - y no solo de la familia - ante el desafío de educar a los niños/as y adolescentes en nuestro tiempo.

Para definir cuáles son estos desafíos, qué se le pide a la educación en nuestro país y en el siglo XXI, es necesario partir de una visión de la educación global y sistémica, que necesariamente afronte la pregunta de la inclusión social y educativa en la era de la sociedad red más allá de la eterna disputa centrada en el currículum escolar (Fernández Enguita, 2011). En el fondo de la cuestión subyace la pregunta sobre cuál debe ser hoy en día el rol de la escuela. Y la respuesta se debe articular desde una comprensión del hecho educativo que permita delimitar qué responsabilidades y funciones corresponden a cada uno de los agentes implicados en la educación, a la vez que simultáneamente se definen los hitos a conseguir conjuntamente y el tipo de gobernanza (Sorensen y Torfing, 2009, 2011) que facilite y estructure el trabajo colaborativo (Milward y Provan, 2006). En esta dirección, investigaciones recientes muestran cómo el abordaje comunitario en educación, con la escuela en el centro, asegura éxitos que no logran las reformas tradicionales (Renée y McAlister, 2011), pues estas tienden a aislar la escuela del resto del sistema económico y político esperando, paradójicamente, que resuelva los problemas sociales (Anyon, 2005).

Reconociendo el interés teórico-práctico de este planteamiento, en este artículo nos proponemos dos objetivos: a) aproximarnos a las bases teóricas que deberían regir las prácticas profesionales del trabajo socioeducativo en red, tanto desde la perspectiva de la acción como desde los retos de su organización, gestión y liderazgo; y b) contrastar este marco teórico con las evidencias que se derivan de su aplicación en 4 experiencias consolidadas y relevantes en Cataluña (provincia de Barcelona). 


\section{EL TRABAJO EN RED Y LA COLABORACIÓN ENTRE INSTITUCIONES EDUCATIVAS Y SOCIALES}

El concepto red - como sistema de conexiones- se ha extendido y popularizado a partir del crecimiento de la informática e internet. Como metáfora, también explica nuevas formas de organizar la relación entre personas e instituciones capaces de superar ciertas barreras espacio-temporales (Edelenbos y Klijn, 2007; Saz, 2007), adquiriendo significados de mayor amplitud cuando plantea alternativas a la gobernanza de los sistemas de bienestar o de la acción educativa, social y cultural (Subirats y Albaigés, 2006; Miller, Díaz-Gibson, Miller-Baslev y Scalan, 2012).

En el mundo educativo y del trabajo social se ha podido constatar en la última década la expansión del concepto y su eclosión en numerosas experiencias de trabajo en red (tabla 1). A nivel internacional destacamos las Zones d'Éducation Prioritaire francesas; las Education Action Zones y Extended Schools del Reino Unido, las Educational Priority Policy belgas, los Kvarter-løft (Neighborhood Lift) daneses, las Area-Based Partnerships irlandesas, las Grote Steden Beleid (Major Cities Policy) holandesas, o los Promise Neighborhoods y las Harlem Children's Zone americanas. Y a nivel nacional constatamos cómo en Cataluña se han generado numerosas experiencias de trabajo socioeducativo en red fruto de iniciativas municipalistas o del propio gobierno autonómico (Blasco y Casado, 2011; Civís, Longás, Longás y Riera, 2007; Fundació Pi i Sunyer, 2011), como es el caso de los Planes Educativos de Entorno, los Proyectos Educativos de Ciudad o las Redes Educativas Locales.

\begin{tabular}{ll}
\hline \multicolumn{1}{c}{ Proyectos } & \multicolumn{1}{c}{ Propósito } \\
\hline $\begin{array}{l}\text { Zones d'Éducation Prioritaire } \\
\text { (Francia) }\end{array}$ & $\begin{array}{l}\text { Planes destinados a fortalecer las relaciones fa- } \\
\text { milia-escuela y abrir la escuela a la comunidad } \\
\text { trabajando conjuntamente en las actividades fuera } \\
\text { del horario escolar. Se implementan en zonas es- } \\
\text { pecialmente carenciadas. }\end{array}$ \\
\hline $\begin{array}{l}\text { Education Action Zones } \\
\text { (Reino Unido) }\end{array}$ & $\begin{array}{l}\text { Planes orientados a coordinar el trabajo conjunto } \\
\text { entre familias, escuela y territorio, dando res- } \\
\text { puesta a necesidades concretas de la comunidad } \\
\text { (absentismo, inserción laboral...). Se dirigen a las } \\
\text { zonas más carenciadas. }\end{array}$ \\
\hline $\begin{array}{l}\text { Extended Schools } \\
\text { (Reino Unido) }\end{array}$ & $\begin{array}{l}\text { Dirigidas al éxito escolar, se trata de escuelas que } \\
\text { asumen la imposibilidad de realizar el trabajo } \\
\text { en solitario y buscan la complicidad de distintos }\end{array}$ \\
& servicios de la comunidad para dar continuidad \\
al trabajo escolar una vez finalizado el horario \\
lectivo.
\end{tabular}




\begin{tabular}{ll}
\hline \multicolumn{1}{c}{ Proyectos } & \multicolumn{1}{c}{ Propósito } \\
\hline $\begin{array}{l}\text { Kvarter-løft } \\
\text { (Dinamarca) }\end{array}$ & $\begin{array}{l}\text { Programa de renovación urbana para mejorar la } \\
\text { calidad de vida de los barrios más deprimidos a } \\
\text { través de proyectos de desarrollo comunitario en } \\
\text { base a la cooperación público-privada }\end{array}$ \\
\hline $\begin{array}{l}\text { Harlem Children's Zone } \\
\text { (EEUU) }\end{array}$ & $\begin{array}{l}\text { Experiencia dirigida a reducir el achievement gap } \\
\text { que se da entre blancos y negros en este barrio de }\end{array}$ \\
& $\begin{array}{l}\text { Nueva York. Provee de servicios sociales, de salud } \\
\text { y educativos de un modo integrado. Cuenta con } \\
\text { programas de parentalidad, de tiempo libre, de } \\
\text { refuerzo escolar, etc. }\end{array}$ \\
\hline $\begin{array}{l}\text { Promise Neighborhoods } \\
\text { (EEUU) }\end{array}$ & $\begin{array}{l}\text { Programa de alcance nacional que replica la expe- } \\
\text { riencia de la Harlem Children's Zone. }\end{array}$ \\
\hline $\begin{array}{l}\text { Planes Educativos de Entorno } \\
\text { (Cataluña) }\end{array}$ & $\begin{array}{l}\text { Planes dirigidos a articular las propuestas educa- } \\
\text { tivas dentro y fuera de la escuela para mejorar el } \\
\text { éxito escolar y la inclusión social y educativa. }\end{array}$ \\
\hline $\begin{array}{l}\text { Proyectos Educativos de Ciudad } \\
\text { (Cataluña) }\end{array}$ & $\begin{array}{l}\text { Planes estratégicos de educación para transformar } \\
\text { la ciudad en un modelo de ciudad educadora. }\end{array}$ \\
\hline $\begin{array}{l}\text { Redes Educativas Locales } \\
\text { (Cataluña) }\end{array}$ & $\begin{array}{l}\text { Redes que articulan entidades y agentes del terri- } \\
\text { torio en base a objetivos socioeducativos compar- } \\
\text { tidos (transición escuela trabajo, éxito escolar). }\end{array}$ \\
\hline
\end{tabular}

Tabla 1. Proyectos socioeducativos comunitarios y/o en red internacionales y nacionales

Fuente: Adaptación y ampliación a partir de Miller, Díaz-Gibson, Miller-Baslev y Scalan (2012)

Seguramente la emergencia de experiencias de trabajo en red indica la búsqueda de respuestas a los retos socioeducativos que plantea una realidad social compleja, diversa y cambiante (Morin, 2004; Castells, 2004; Bauman, 2007). Una realidad que desborda la estructura de servicios a las personas creada en los estados del bienestar y que requiere prácticas organizativas y profesionales diferentes a las aplicadas hasta la fecha (Waissbluth, 2008). Concretamente, en el sector socioeducativo constatamos cómo el modo con el que se ha venido operando no es suficiente o incluso no es válido. Este a menudo plantea respuestas simples para problemas complejos (Barnett y McCormick, 2012; Longo, 2008; Ubieto, 2009, 2012) hecho que, en el mejor de los casos, resuelve situaciones puntuales y en el peor, no resuelve o empeora. El resultado de la lógica aplicacionista y la organización de los servicios a las personas desde la especialización por disciplinas científicas ha construido una cultura profesional actualmente cuestionada. Responder a las necesidades sociales con estrategias y acciones específicas, focalizadas en el "problema» y excesivamente disgregadas, segmentadas o silo thinking (Eggers y Singh, 2009; Miller y Jæger, 2011), genera soluciones insuficientes por ser una forma parcial y restringida de abordar desafíos complejos (Morin, 2004; Vilar, 2008). 
Y si nos referimos explícitamente a la escuela, debemos reconocer que hace demasiado tiempo que sufre los efectos de actuar ante la complejidad educativa de modo simplificador e incluso gremial. Sabemos que el momento actual es muy exigente para la escuela, no solo por los retos que la sociedad del conocimiento y la globalización conllevan, sino también por el incremento de demandas que la familia y la sociedad en su conjunto le transfieren. Ante ello, la escuela está colapsada, adolece de falta de recursos y ve reducida su capacidad para cubrir a plena satisfacción las funciones que la sociedad le encomienda.

Por ello resulta lógico la emergencia de proyectos de base comunitaria que, mediante la colaboración entre instituciones, entidades y profesionales, buscan dar respuestas integrales a los problemas educativos compartidos (Subirats y Albaigés, 2006; Miller, Díaz-Gibson, Miller-Baslev y Scalan, 2012). Se trata de iniciativas en las que participan de forma estable, aunque sea con desigual intensidad, los distintos agentes socioeducativos de un territorio (escuelas, técnicos municipales de educación, servicios sociales, promotores de empleo, entidades del tercer sector, etc.) para articular la colaboración entre ellos y, de este modo, mejorar los resultados educativos y la protección e inclusión social de niños y adolescentes. Estas nuevas formas de organización interinstitucional e interprofesional, muchas en plena fase de experimentación, frecuentemente se han autodefinido como redes (redes locales o territoriales), dando a entender que pretenden superar las clásicas comisiones de coordinación para abrirse a alianzas estables en pos de un proyecto compartido.

Si partimos de un primer concepto genérico que define red como una estructura (organización) formada por diferentes nodos conectados entre sí, podemos definir las redes socioeducativas locales (RS) como un modelo de organización alternativo a los tradicionales organigramas jerárquicos, con capacidad para integrar instituciones y actores con responsabilidad educativa en un plano de corresponsabilidad, unidos por el interés común, primero para compartir el análisis de las necesidades y los proyectos de un territorio o comunidad, y segundo para coordinar la acción socioeducativa de un modo integral y coherente (Longás, Civís y Riera, 2008, p.306). Se trata de estructuras que promueven la conexión de los distintos agentes socioeducativos para favorecer el intercambio de información, la coordinación y la toma de decisiones entre sus miembros en relación a los objetivos comunes. Y, en la medida que son explícitas y responden a ciertos grados de formalización, son formas consistentes de organizar la colaboración en la acción socioeducativa a partir de la interdisciplinariedad e interinstitucionalidad.

En el sentido apuntado, podemos considerarlas redes de conocimiento, dado que el intercambio de información y las interacciones estables entre 
los distintos agentes generan nuevos productos o resultados (Díaz, Civís, Longás y Murat, 2010). Entre ellos, y de suma importancia, la elaboración de un "relato» común sobre las situaciones en las que se trabaja y la forma de abordarlas, así como una renovada concepción de la acción profesional. El enraizamiento de las redes en el territorio - próximo a personas, instituciones y profesionales - facilita que los agentes socioeducativos compartan la comprensión de las necesidades, la visión respecto a las soluciones y el empeño en optimizar las respuestas partiendo de los recursos existentes y generando nuevas acciones.

\section{PRINCIPIOS DEL TRABAJO SOCIOEDUCATIVO EN RED Y GESTIÓN DE LAS REDES}

En anteriores investigaciones hemos reconocido la existencia de seis principios que determinan la madurez o calidad de las RS (Longás y Civís, 2010; Díaz y Civís, 2011). A continuación los definimos brevemente:

- Proximidad: representa la exigencia de responder a necesidades reales del territorio. Las redes locales tienen su punto de arranque en el conocimiento y reconocimiento del propio entorno, se crean en contextos determinados y se caracterizan por la contextualización de sus acciones. Tanto el reconocimiento profesional como la identidad de las personas y agentes educativos que integran una comunidad son elementos clave para impulsar el trabajo socioeducativo en red evitando caer en acciones tecnocráticas.

- Transversalidad: comporta la visión integral —e integradora- de la acción por parte de las diversas instituciones o actores que forman la RS. Ello implica reconocer la complejidad de los fenómenos sociales y aproximar las miradas e intereses puramente sectoriales y/o institucionales hacia un interés conjunto, multidisciplinar, que debe ser abordado transversalmente. Cuando se sitúa al ciudadano y sus necesidades en el centro, el principio de transversalidad emana de forma espontánea. Es una respuesta integradora y coherente en tanto que aporta una optimización general de los recursos alejándose de la segmentación y la departamentalización de las respuestas o de la acumulación y solapamiento de acciones que habitualmente genera el trabajo en paralelo.

- Horizontalidad: se refiere al tipo de estructura y relación que caracteriza a la organización y formalización de la red. El trabajo transversal se desarrolla en un plano de igualdad en el cual todos los agentes pueden interaccionar en un mismo nivel jerárquico. La organización 
se extiende horizontalmente para facilitar la visión integral y la acción conjunta respecto a las necesidades, la definición de objetivos, el plan de trabajo y la metodología a seguir. La organización no jerárquica de las RS requiere un liderazgo compartido, no asociado a un solo nodo.

- Corresponsabilidad: inspira el trabajo en red, sustenta la colaboración de sus miembros y debe promover la armonización de sus intereses. Los distintos actores de una red tienen distintas responsabilidades y roles, y la red permite el trabajo conjunto desde el reconocimiento mutuo y el compromiso compartido.

- Colaboración: incide sobre las dinámicas de relación entre las diversas instituciones y actores. Contrariamente al hermetismo institucional, una estructura relacional interdependiente sitúa la colaboración como condición fundamental para el éxito. La transversalidad de las necesidades y objetivos, la horizontalidad y la responsabilidad compartida, precisan dinámicas de interacción en las que cada actor aporta su experiencia y perspectiva para poder construir una respuesta verdaderamente global. Los miembros de una red han de entender que comparten un mismo problema y que cada uno puede aportar un fragmento de respuesta, de modo que la respuesta nunca será completa sin la colaboración de los demás (Subirats y Albaigés, 2006).

- Proactividad y proyección: enfatiza la importancia de la planificación estratégica en el trabajo en red y su orientación al logro u obtención de resultados. La red, para sustentar una acción conjunta, continua y en cierta forma sostenible, no debe centrarse tanto en su dimensión operativa como en la capacidad para pensar estratégicamente, lo que implica actuar preventivamente y establecer los canales de solución de futuros problemas (Collet, 2009). Este principio supone trabajar con una metodología de acción-reflexión que permita evaluar el proceso y los resultados, mejorando la comprensión del hecho socioeducativo y afinando progresivamente su abordaje.

Ante la complejidad inherente de las RS, derivada de las necesidades de concertación de profesionales de distintos sistemas y con culturas —organizativas y profesionales - diferentes, estos principios orientan su gestión hacia un funcionamiento más efectivo que fortalece el capital social, como outcome resultante de su acción, y que deviene eficiente en la consecución de sus objetivos (Ubieto, 2007; Klijn, Edelenbos y Steijn, 2010; Díaz y Civís, 2011). En sí mismos suponen una guía para desarrollar una cultura profesional ampliada (Waissbluth, 2008), capaz de orientarse holísticamente y desde la colaboración interprofesional, y su cumplimiento indica un mayor grado de madurez y calidad de las RS y el trabajo socioeducativo. En la 
figura 1 se esquematiza una propuesta de gestión integrada de los 6 principios, de modo que algunos responden a las necesidades de obtención de resultados mediante la adecuada ejecución u organización de la acción (línea), mientras que los otros responden a las necesidades más consultivas o reflexivas (staff) de la organización, vinculados a estructuras facilitadoras de la participación y vinculación de los miembros. El crecimiento de estos ejes, convertidos en lados de un paralelogramo, se apoya y favorece un mejor logro de los objetivos compartidos, el fortalecimiento de la cultura colaborativa y el desarrollo del capital social (eje diagonal o resultante).

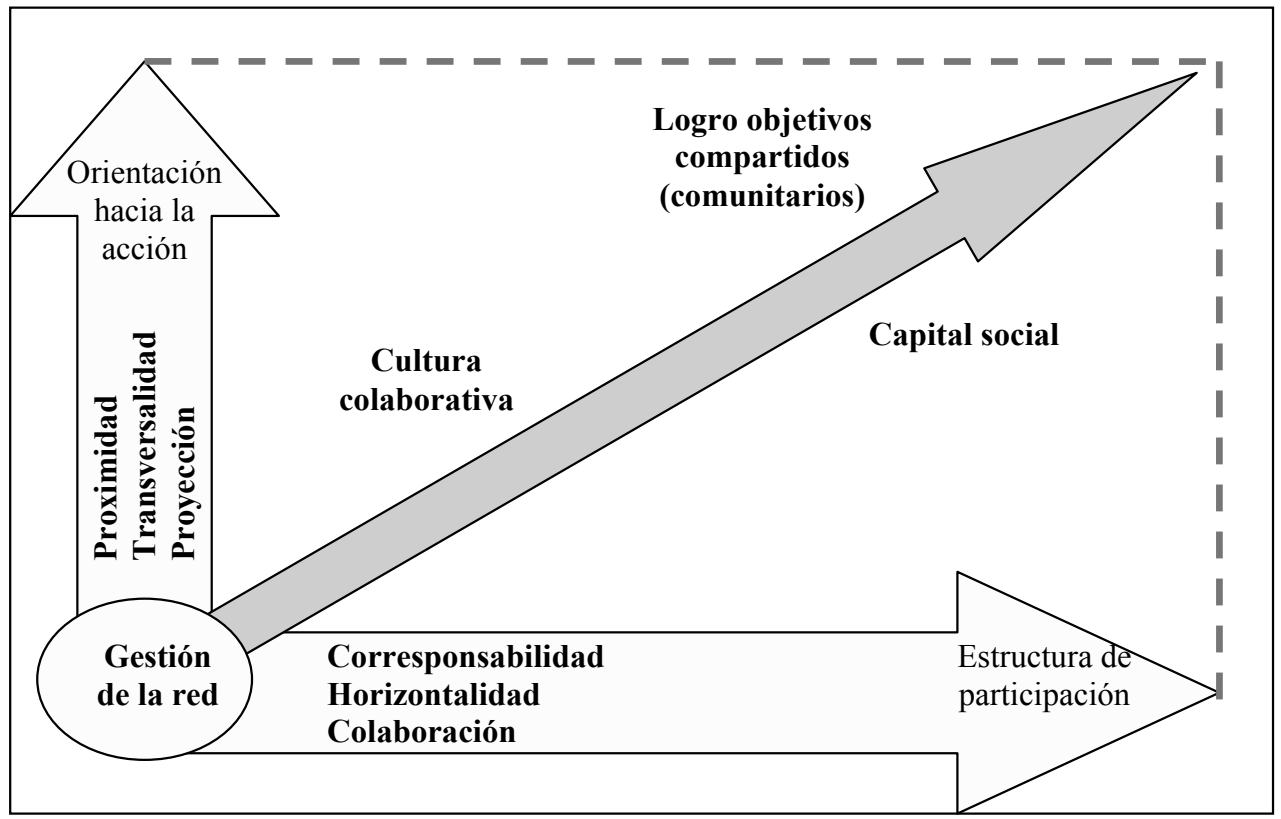

Figura 1. Propuesta de gestión integrada de las redes socio-ducativas

Fuente: Reelaboración a partir de Díaz-Gibson, Civís y Longás (2012)

Para gobernar las redes de modo eficaz y eficiente según los principios de calidad presentados, las funciones de gestión y liderazgo son determinantes. Milward y Provan (2006) identifican cinco tareas clave que satisfacen las necesidades de gestión de las redes y caracterizan su buen liderazgo: la gestión del rendimiento de cuentas, de la legitimidad, del conflicto, del diseño y del compromiso. Para Hinterhuber (2002) las cuatro funciones características del network orchestrator, que es como denomina al gestor de redes, son las de arquitecto, juez, promotor y líder. Según este autor, ser competente en estas tareas o funciones es fundamental para que la red avance y se consolide. A continuación, integrando ambas aportaciones, las explicamos brevemente. 
- Rendimiento de cuentas (Milward y Provan, 2006) o función de juez (Hinterhuber, 2002): dado que la organización no es jerárquica, es imprescindible determinar las responsabilidades (quién responde de qué). Garantizar la continuidad de una red precisa definir estándares de rendimiento y a su vez rendir cuentas, así como proveer formas de control sobre las tareas asignadas o distribuidas. Igualmente, tiene sentido poner de manifiesto las aportaciones de cada miembro para contribuir a su reconocimiento.

- Legitimidad: es un asunto mucho más complicado para las redes que para las organizaciones clásicas dado que estas responden a una misión o finalidad que las legitima internamente desde su creación (Milward y Provan, 2006). En cambio, una red necesita constantemente negociar y probar su legitimidad para conseguir que sus miembros den sentido a su continuidad, a seguir contribuyendo y cooperando. La reputación, la aceptación y el reconocimiento social acabarán siendo las claves de su legitimidad, por lo que la gestión deberá invertir en capitalizar logros tangibles, dar visibilidad a la red, incorporar nuevos miembros, ampliar su radio de acción, etc.

- Gestión del conflicto: el conflicto suele aparecer cuando hay diversidad de objetivos y prioridades por parte de los miembros de la red (Milward y Provan, 2006), cuando no se reconocen y armonizan las diversas aspiraciones legítimas derivadas de las adscripciones institucionales de cada miembro y cuando las maneras de funcionar son muy distintas. Será fundamental que el liderazgo ayude a orientar la gestión del conflicto constructivamente y en base al diálogo para aprender y avanzar, negociando prioridades y objetivos compartidos.

- Gestión del diseño y organización de la red (Milward y Provan, 2006) o función de arquitecto (Hinterhuber, 2002): es necesario articular adecuadamente la red en función de su tamaño y biografía para obtener un buen control de la acción y alcanzar resultados. Una red pequeña o en su fase inicial podrá operar fácilmente en base a la construcción de consensos, mientras que una red que incorpora a muchos actores necesitará una estructura organizativa que combine la representatividad y la capacidad operativa para evitar que la necesidad del consenso se convierta en un freno constante. Se reconocen tres tipos de estructuras: autogobierno, con una entidad o agente que lidera, y con el liderazgo distribuido.

- Gestión del compromiso (Milward y Provan, 2006) o funciones de promotor y líder (Hinterhuber, 2002): es determinante la motivación y desarrollo de activos físicos e intelectuales para sostener las redes. 
El salario de los miembros de una red lo pagan las respectivas organizaciones y, en consecuencia, dependen y están comprometidos en primera instancia con la institución que los contrata. Resulta fundamental para el éxito de la red que sus miembros encuentren sentido a la red y al rol que desempeñan, que se impliquen y participen. Pero también que las distintas instituciones reconozcan y apoyen este trabajo. Debemos apuntar el acierto que supone como parte de la gestión del compromiso la institucionalización de algunas relaciones que pueden considerarse clave asegurar la sostenibilidad de la red por encima de personalismos o vínculos particulares. Las redes con cierta madurez tienden a mayor grado de formalización.

Conviene destacar que estas tareas y funciones deben ser reinterpretadas internamente en el seno de cada una de las organizaciones en red. No debemos olvidar que el estilo del liderazgo, su ejemplo, el modo de gestionar y el filtraje de actitudes y aptitudes condicionan el conjunto de la red tanto en referencia a la propia gestión como al nivel de confianza y al clima generado, siendo determinante de su éxito (Waissbluth, 2008).

En síntesis, el trabajo socioeducativo que responde a un modelo de red de conocimiento debe regirse por unos principios que articulan y organizan la acción y requiere de liderazgos sofisticados, capaces de responder a aquellos aspectos específicos y críticos que condicionan la existencia y desarrollo de la red - como realidad en continuo proceso de construcción-y al avance de las acciones previstas orientadas al logro de los objetivos planteados.

\section{ANÁLISIS DE BUENAS PRÁCTICAS DE TRABAJO INTERINSTITUCIONAL EN RED}

El Área de Educación de la Diputación de Barcelona inició en el año 2000 una decidida política de apoyo a la inclusión social y educativa a nivel local mediante el estímulo de procesos de trabajo conjunto entre los técnicos y servicios municipales, los centros y servicios educativos, las entidades del tercer sector y otros actores implicados en la educación (Bosch, Bravo, Alonso et al., 2008). A partir del lema Red de Municipios, este organismo que agrupa 311 municipios ( $75 \%$ de la población catalana) ha impulsado el trabajo en red para fortalecer principalmente los proyectos educativos de ciudad, la atención a la infancia, la mejora del éxito escolar y la transición escuela trabajo (TET). Fue a raíz de estas experiencias que en el año 2006 el Departament d'Ensenyament creó los Planes Educativos de Entorno (PEE), legitimando al máximo nivel las estrategias de trabajo interinstitucional en red para mejorar el éxito escolar y la inclusión educativa (Generalitat de Catalunya, 2012). 
A continuación describimos 4 experiencias exitosas de trabajo interinstitucional en red en nuestro contexto catalán. Posteriormente, a partir de su comparación y según el marco teórico presentado, pretendemos establecer algunas conclusiones preliminares que puedan ayudar a su fortalecimiento o incluso a su transferencia a otros contextos. Concretamente hemos seleccionado las experiencias de l'Hospitalet de Llobregat, Olesa de Montserrat, Badia del Vallès y Sant Vicenç dels Horts que fueron consideradas buenas prácticas en un reciente seminario dedicado a las RS organizado por la FPCEE Blanquerna (Longás y Civís, 2009) por las siguientes razones: a) están consolidadas (son estables), b) obtienen buenos resultados, c) se ubican en contexto metropolitano, d) se han generado desde la iniciativa local, y e) en su inicio el foco ha sido la mejora de la inclusión social y educativa de adolescentes y jóvenes, con énfasis en los procesos de transición escuela trabajo (TET). Para cada una de ellas organizamos la información en los siguientes apartados: historia (origen, desarrollo y tendencia de evolución), contenido (finalidad y objetivos que persigue), organización (agentes implicados, estructura y liderazgo) y logros (resultados de proceso y de impacto).

\section{Dispositivo Local de Inclusión y Acompañamiento Escolar en Hospitalet de Llobregat}

Historia: La prevención del absentismo escolar ha sido y es un eje prioritario del trabajo educativo en la ciudad. En el año 2001, buscando atajar el absentismo en $3 .^{\circ}$ y $4 .^{\circ}$ de ESO, se realizó el pilotaje del Programa de Orientación de Alumnos, en colaboración con los 4 institutos de una zona educativa del municipio. Se trabajó para mejorar la prevención, la orientación y la formación de jóvenes con dificultades de aprendizaje. El exitoso trabajo conjunto entre institutos, entidades sociales y centros de formación no reglada permitió impulsar posteriormente un PEE y generalizar la experiencia a todos los centros.

Contenido: El objetivo principal del dispositivo (DLI) es reducir el absentismo y mejorar el acompañamiento de los alumnos con dificultades en su escolarización. Se busca mejorar el éxito escolar mediante la acción preventiva y el trabajo colegiado. Han sido objetivos y criterios compartidos: reducir obstáculos, construir nuevas formas de acompañamiento, estimular la pedagogía del pacto y el compromiso y trabajar con las familias.

Organización: Actualmente el DLI se desarrolla en el marco del PEE. Dicho plan ha supuesto un nuevo escenario para impulsar el trabajo en red entre el equipo técnico del área de educación del ayuntamiento, los servicios sociales, los centros educativos y las entidades locales. La coordinación general se hace en la mesa educativa. Existen comisiones socioeducativas en 
cada centro educativo (referentes del centro educativo, Equipo de Atención Psicopedagógica, Servicios Sociales y DLI). Los casos son detectados en el centro y la comisión elabora un plan de intervención personalizado.

Logros: La implementación del PEE ha sido la evolución de la experiencia inicial de reducción del absentismo escolar y mejora del acompañamiento de los alumnos con dificultades TET. La organización y extensión del DLI es en sí mismo un notable resultado de proceso. También se observan cambios cualitativos en la percepción y atención de los alumnos con dificultades por parte de los docentes, más abiertos a confiar en sus posibilidades y promover las oportunidades de inclusión educativa. Finalmente, destacar la reducción casi total del absentismo escolar y la elaboración de planes individuales de acompañamiento para la mayoría de alumnos con riesgo de abandono escolar.

\section{Programa de Transición Escuela-Trabajo de Olesa de Montserrat}

Historia: En el curso 2005-2006 se crea la comisión TET para responder a los elevados índices de desempleo juvenil, fracaso escolar y absentismo a finales de la ESO. Con el asesoramiento facilitado por la Diputación se inicia una estrategia global de acción interinstitucional. En 2011 se realiza una diagnosis socioeducativa y en 2012 se diseña el proyecto de RS, buscando la implicación de todos los actores socioeducativos que inciden en las etapas 0-18 años.

Contenido: La finalidad del programa inicial es mejorar los procesos TET. Las acciones atienden 4 líneas de intervención: prevención (mejorar del éxito escolar, la formación profesional inicial y el desarrollo de competencias profesionales en la ESO), orientación y acompañamiento (final de ESO y durante el proceso de transición), formación (mejorando las opciones de formación profesional no reglada) e inserción (aumentando las oportunidades para acceder al trabajo de los jóvenes). Se ha formalizado un protocolo de derivación y seguimiento de los jóvenes con necesidades TET que asegura el acompañamiento a lo largo de todo el proceso.

Organización: lidera un equipo del ayuntamiento formado por las áreas de promoción económica, educación, juventud y servicios sociales. Existe una comisión TET que gestiona directamente las acciones planificadas para el curso, integrada por la técnica del dispositivo TET (Promoción Económica), responsables psicopedagógicos de los institutos, técnico del Equipo de Atención Psicopedagógica de la zona y el educador social del municipio. Se ha dotado al programa de una técnica que coordina el dispositivo TET y realiza el acompañamiento de los jóvenes fuera de la escuela. 
Se dispone de un observatorio que informa cada curso de los resultados obtenidos y su tendencia.

Logros: Como resultados de proceso: consolidación del equipo interdepartamental en el ayuntamiento que actúa como grupo motor del programa TET y del trabajo en red; alta valoración de alumnos y centros de las acciones TET; creación del Programa de Formación y Aprendizaje Profesional; creación del Proyecto de Responsabilidad Compartida «Teu» para potenciar la acreditación de la ESO; y el plan específico para la intervención con familias. En su impacto, se aprecia la tendencia a la reducción del fracaso escolar en la ESO y la mejora significativa en la cualificación, formación e inserción de los jóvenes con necesidades TET. Los resultados de inserción se han reducido a raíz de los efectos de la crisis económica sobre el empleo.

\section{Programa Integral de Acción Socioeducativa de Badía del Vallés}

Historia: La Generalitat de Catalunya concede al municipio un PEE en el año 2005. Su puesta en marcha, desde el Área de Educación y el Área de Juventud, activa a diferentes actores del municipio. Se realiza una diagnosis participativa y se organizan diversas comisiones, siendo la Comisión TET la más diligente en funcionar y obtener los primeros resultados. Posteriormente se elabora un plan estratégico de mejora socioeducativa que cristaliza en un Plan de Inclusión (Departament de Benestar Social i Família de la Generalitat de Catalunya). Gracias a la legitimación del proceso y el reconocimiento de la transversalidad se inicia la progresiva redefinición de la estructura organizativa de los servicios municipales de atención a las personas, desembocando en una programación unificada y una sola unidad de gestión. El proceso se apoya en la creación y mantenimiento de una RS.

Contenido: El modelo consiste en gestionar todos los programas socioeducativos desde su integración en un plan conjunto y unificado de todas las áreas del ayuntamiento. Se parte de una planificación de objetivos socioeducativos, con sus correspondientes acciones y recursos, desde una perspectiva integradora y a partir de 6 ejes de trabajo compartidos y consensuados por los distintos profesionales: apoyo a la familia, habilidades para la vida, formación, salud, convivencia-modelos de relación y participación-red social-ocio. A su vez, el programa común considera 4 segmentos de población diana: maternidad y pequeña infancia, infancia, adolescencia y juventud. La intersección de ambos ejes crea una matriz de trabajo transversal que permite realizar y actualizar un diagnóstico compartido en torno a qué necesidades concretas se encuentran en el territorio, integrando las miradas de los profesionales de los diversos actores. La realización de este diagnóstico permite obtener un cuadro de necesidades socioeductivas del 
territorio relativas a los diferentes segmentos de población, analizar los recursos y respuestas que se están dando, priorizar su atención y, finalmente, estructurar la planificación de tareas.

Organización: Se ha tendido a la disolución de los departamentos municipales entendidos como tales, aunque existen formalmente dado que no se ha eliminado la estructura administrativa. Se ha creado una gran Área de Servicio a las Personas que agrupa a todos los técnicos municipales de Educación, Servicios Sociales y Juventud en una sola unidad de acción. El liderazgo recae sobre la figura de un coordinador para toda el área. Los presupuestos están unificados y se trabaja por programas, a los que se asocian equipos en función de los objetivos y especialización profesional. También se ha reorganizado el espacio de trabajo hasta conseguir una «oficina única». El trabajo de la RS, apoyado liderado desde esta estructura, permite articular la colaboración con los centros educativos y las entidades locales. La red se organiza mediante una mesa plenaria y comisiones.

Logros: El principal es el propio Programa Integral de Acción Socioeducativa que propone 13 ámbitos de trabajo o subprogramas: Vínculo familiar, Redes familiares, Familia y escuela, Habilidades para la vida, Comunicación e información, Mejora de oportunidades educativas, Salud, Convivencia, Género, Diversidad, Relaciones interprofesionales, Ocio y Participación. En él se establecen los objetivos y acciones, así como las conexiones entre subprogramas y los indicadores y estándares de evaluación. Las acciones que operativizan el programa no tienen por qué pertenecer a un servicio o departamento concreto, siendo indiferente la pertenencia al servicio de «origen», a la vez que incluye acciones que desarrollan otros servicios y entidades. El programa deviene el cuadro de mando de la acción socioeducativa del ayuntamiento y aspira a convertirse en el relato de dicha acción en el ámbito local.

\section{Red Socioeducativa de Sant Vicenç dels Horts}

Historia: Nace el año 1999 de modo espontáneo, gracias a la coincidencia de los 3 institutos, la Inspección de Educación y la técnica de la Concejalía de Educación. Poco después se adscribe a las primeras experiencias de trabajo en red que impulsa la Diputación de Barcelona. Inicialmente se atienden las significativas necesidades TET del municipio y se generan estructuras de trabajo transversal entre distintas concejalías municipales (Educación, Servicios Sociales, Trabajo y Juventud), así como una mesa estable de trabajo con los institutos y entidades locales. La concesión y posterior desarrollo de un PEE permite ampliar la red (incorporación de centros de primaria, asociaciones de padres, área de deportes, etc.) y ampliar 
el foco de actuación, contemplándose objetivos más ambiciosos de mejora educativa en general (Longás, Fontanet y Bosch, 2007).

Contenido: Se orienta a la mejora del éxito escolar y la inclusión social de la infancia y la juventud a partir de los ejes: éxito escolar, entorno educativo y transición entre etapas educativas (con especial énfasis en la TET). Desde la red se pretende mejorar la coordinación, la calidad y el compromiso en las acciones de todos los actores y ampliar las oportunidades educativas. A su vez se valora de forma explícita la importancia del proceso realizado, de modo que se reconocen también como objetivos del trabajo en red la necesidad de: a) relacionar los distintos actores que intervienen en los procesos de educación formal para favorecer la colaboración y el trabajo conjunto, b) analizar conjuntamente las necesidades educativas y las respuestas que se articulan, c) disponer de espacios de coordinación profesional e interinstitucional, y d) disponer de estructuras locales de empoderamiento que permitan acceder a mayor conocimiento y a mayores recursos de los miembros de la red.

Organización: Se organiza una plataforma plenaria o mesa local que agrupa todos los agentes socioeducativos que intervienen de forma directa o indirecta en la educación (centros educativos de primaria y secundaria; escuela de adultos; servicios locales de Educación; Inspección Educativa; técnicos municipales de educación, juventud, servicios sociales, trabajo y deportes; entidades de acción socioeducativa; asociaciones de padres y madres; y asesores de la universidad). A su vez existen 4 comisiones que funcionan de forma continua todo el curso: comisión operativa o de gestión, mejora del éxito escolar, transición escuela-trabajo y entorno e inclusión. La técnica de educación es responsable de la comisión operativa y desarrolla una clara función de liderazgo como secretaria general de la mesa educativa. Las otras comisiones y proyectos concretos cuentan con funcionamiento autónomo y liderazgos propios, rindiendo cuentas a la comisión operativa de modo más continuo y a la mesa plenaria cuando se diseñan los planes de trabajo o se realiza su evaluación. Se dispone de una planificación estratégica realizada a partir de un mapeo inicial de necesidades y recursos, y se gestiona anualmente mediante planes, presupuestos y evaluación de procesos y resultados.

Logros: dispositivo local de inserción para jóvenes; informe sobre necesidades psicosocioeducativas de los alumnos de primaria y secundaria; observatorio TET; catálogo de recursos socioeducativos para adolescentes y jóvenes; plan de dinámica educativa; grupos de estudio asistido; programas de aprendizaje y servicio; plan de práctica deportiva para todos; jornadas formativas y de intercambio de buenas prácticas para docentes y otros profesionales vinculados al PEE; boletín informativo y página web; y la partici- 
pación en redes a nivel supramunicipal. Como principal impacto destaca la significativa reducción del fracaso escolar en cerca de 15 puntos (se partía de 33\% de no acreditación a inicios de la década 2000-2010).

\section{Valoración y discusión de las experiencias}

Las experiencias descritas ilustran estrategias de colaboración interinstitucional orientadas a la inclusión (social y educativa) especialmente focalizadas en la mejora del éxito escolar. Todas ellas se iniciaron para dar respuesta a necesidades socioeducativas de los adolescentes que finalizaban la ESO sin éxito (procesos TET) y han evolucionado, aunque con ritmos y procesos diferentes, en una dirección equivalente: consolidando el trabajo en red, ampliando los objetivos y/o foco de la intervención y aumentando los agentes socioeducativos que participan de este trabajo. Si consideramos que en todos los casos la adhesión de los miembros a la RS tiene un escaso o nulo nivel de reconocimiento (las instituciones y entidades que integran las redes no suelen considerar el trabajo interinstitucional en su manual de funciones y política salarial), la consolidación y progresiva formalización o institucionalización de estas experiencias de colaboración indica, en nuestra opinión, su valor e interés para los profesionales.

La evolución y fortaleza de estas experiencias, que han superado diversas dificultades de gestión, financiación e incluso en algún caso de presiones políticas, apunta hacia la validez de los principios que según el modelo teórico definen la madurez de una RS. Y pensamos que dicha madurez se sustenta no tanto en la intensidad como se satisfacen algunos de ellos sino en su gestión acompasada e interrelacionada (Longás y Civís, 2010; Díaz y Civís, 2011). Lo discutimos a continuación.

El sustrato de las 4 experiencias es la actitud de corresponsabilidad y compromiso con un proyecto socioeducativo común, generado desde el mundo local para atender necesidades socioeducativas concretas detectadas en y desde el propio territorio (proximidad). De algún modo, el conocimiento compartido genera un tipo de comprensión holística del fenómeno social y educativo que empuja a los distintos actores a mirar más allá de sus propias organizaciones (de las que dependen jerárquicamente y que tienen sus legítimos requerimientos) para comprometerse con un proyecto de bien común.

Desde el inicio de las 4 experiencias de trabajo en red y en sus posteriores dinámicas de desarrollo, vinculado a mayores logros, está presente el esfuerzo por atender al principio de transversalidad. En todos los casos, los miembros de las primeras comisiones y de las RS consolidadas son de diferentes organizaciones y niveles de la administración pública (inter- 
institucionalidad) y de distintas formaciones y subsistemas profesionales (interprofesionalidad y multidisciplinariedad), de modo que se refuerza el conocimiento y confianza mutua, la colaboración y su interdependencia. El trabajo continuado conjunto, además de favorecer las necesarias coordinaciones de los procesos comunes, teje un entramado de alianzas, relaciones y conexiones que lleva a abordar el reto educativo desde una perspectiva más comunitaria.

Como exigencia común, la narración de los líderes o promotores de estas redes señala que organizar la transversalidad de forma efectiva requiere la búsqueda de mayor participación y la toma de decisiones en base al consenso, contando con estructuras organizativas que lo permitan (horizontalidad). El esfuerzo y tiempo que todo ello precisa fácilmente se ve limitado por la colisión o competencia con las obligaciones que deben cumplir los distintos miembros de las RS con sus respectivas organizaciones.

Sin desconsiderar estas limitaciones, la consolidación de las experiencias estudiadas se ha sustentado en la construcción de una misión común, su proyección en acciones claramente dirigidas a la resolución de las necesidades y la obtención de resultados (proactividad y proyección) en la medida que retroalimentan de forma positiva el trabajo en red.

Destacamos el rol relevante de la escuela como agente clave de las RS. Su participación es indispensable dada su sólida tradición y conocimiento, su especificidad educativa y la universalidad en la atención a la infancia y adolescencia que garantizan. Pero su función en la red no es ejercer el liderazgo o la tutela. La respuesta a los retos de la educación ya no es patrimonio exclusivo de ningún agente para pasar abiertamente a ser de todos. De modo que esta nueva realidad, aunque complique la articulación de las respuestas, exige nuevas culturas profesionales y nuevos modos de organizarse. Y la organización en red, por abordar de forma sistémica el fenómeno educativo, da cabida a todas las voces y miradas en la medida que se evitan subordinaciones entre los miembros implicados.

Finalmente es preciso analizar la gestión y el liderazgo que ha guiado las experiencias descritas y su hipotética relación con los diversos niveles de madurez en el proceso de articulación del trabajo en red. En todas ellas se reconoce un notable grado de éxito en el desempeño de estas funciones críticas en las RS (Milward y Provan, 2006; Waissbluth, 2008) aunque en las dos que actualmente se observa mayor vitalidad y amplitud de acción (Sant Vicenç del Horts y Badía del Vallés) se han dado, al menos, tres circunstancias comunes: a) liderazgo estable y decidido orientado desde el principio hacia el modelo de trabajo en red; b) capacidad para organizar la participación y gestionar los consensos en el análisis de necesidades, toma de deci- 
siones y planificación estratégica, y c) habilidad para buscar sinergias entre los miembros de la red y distribuir el liderazgo entre ellos. En las 4 redes se reconoce como fortaleza el compromiso de las personas responsables de su promoción y liderazgo.

Los resultados más tangibles, aunque en grados diferentes, han evidenciado que la dinamización y organización de la colaboración interinstitucional mediante el trabajo en red ha servido para incrementar la eficiencia y eficacia de los recursos locales. Ello se debe tanto a los efectos de las sinergias entre los miembros de las RS y al rediseño de algunos servicios y acciones que ya estaban presentes en el territorio, como a las formas de seguimiento de las tareas determinadas y asignadas que han impuesto los respectivos network orchestrator (Hinterhuber, 2002). En conjunto todas las experiencias tienen el reconocimiento social que las legitima, gestionan de forma aceptable los conflictos que habitualmente surgen por las propias características complejas de la estructura y de los objetivos que persiguen, y cuentan con diseños ajustados a sus características.

\section{CONCLUSIONES}

Como conclusiones de este trabajo, y por lo tanto de carácter preliminar, las coincidencias entre las 4 experiencias estudiadas apuntan hacia la validez de los principios para la gestión de las RS. La aproximación crítica e investigativa sobre las RS nos indica que estas solo funcionan cuando están bien edificadas, la comunicación está articulada, la toma de decisiones es conjunta y se orientan a mejorar la realidad existente, de modo que ajustan sus planes de acción a las necesidades y demandas reales.

En las experiencias de éxito sobresale la importancia de que las estructuras, la gestión y el liderazgo se orienten hacia la transversalidad y la colaboración. Las RS, como modelo para desarrollar la corresponsabilidad entre instituciones y agentes socioeducativos, solo pueden ser exitosas y sostenibles si superan los desafíos de construir una nueva cultura profesional y alcanzar logros mediante una gestión efectiva.

Hemos señalado por qué determinados modos tradicionales de abordar las necesidades socioeducativa pueden resultar insuficientes. La complejidad de muchos de estos desafíos emergentes —el éxito escolar, la inclusión o la transición escuela-trabajo son buenos ejemplos- requiere articular respuestas desde la suma de visiones, conocimientos, capacidades y recursos de las instituciones y profesionales que los abordan. Si los retos son complejos las respuestas no pueden ser simples o fragmentadas y, en este sentido, las estructuras en red permiten organizarlas desde una visión holística y 
sistémica de la realidad. Es difícil imaginar que el trabajo social y educativo en solitario, «departamentalizado», «uni»-disciplinar o «uni»-institucional pueda tener en el futuro demasiados éxitos, aparte de ciertos resultados puntuales.

En este sentido, debería abandonarse la idea que considera la escuela como el principal agente que responde a los retos que se plantean a la educación y que las problemáticas sociales deben ser resueltas por los servicios profesionales específicos. La escuela tiene una misión muy clara y un componente de especificidad que nadie puede sustituir, pero solo desde un trabajo compartido y corresponsable será posible que dicha especificidad no se malogre por un exceso de demanda y delegación. Respecto al rol de la escuela en las RS, en la praxis hemos encontrado dos modelos no deseables: a) la escuela que lo centraliza e intenta asumir todo, y b) los proyectos socioeducativos que se crean al margen de la escuela, dando el protagonismo a otros agentes socioeducativos y relegando a la escuela a un papel marginal. Por consiguiente, es importante que los diversos agentes socioeducativos, escuela incluida, entiendan la importancia del trabajo compartido, redistribuido y democratizado para atender los desafíos comunes.

Sabemos que existen muchas otras experiencias alrededor de las necesidades educativas emergentes (inclusión e interculturalidad, atención a la primera infancia, educación con/de familias, menores vulnerables, etc.) que están avanzando en diversos territorios gracias al trabajo colaborativo apoyado en la organización de redes. Aunque no han sido objeto de nuestro análisis, y en ello debemos reconocer una limitación de nuestro estudio, la eclosión de esta multiplicidad de iniciativas «multiagentes» parece indicar que asistimos a la emergencia de un nuevo modelo o quizás paradigma de la acción socioeducativa. En este sentido, los aprendizajes y reflexiones que se derivan de este artículo y que sin otras investigaciones que los refuercen no podemos presentar como generalizables, pueden ser útiles para orientar la acción-reflexión de investigadores y gestores del trabajo en red en el campo de las ciencias sociales. 


\section{REFERENCIAS BIBLIOGRÁFICAS}

Anyon, J. (2005). Radical Possibilities: Public Policy, Urban Education and New Social Movement. New York: Routledge.

Barnett, K. y McCormick, J. (2012). Leadership and team dynamics in Senior executive leadership teams. Educational Management Administration \& Leadership, 40(6), 653-671.

Bauman, Z. (2007). Els reptes de l'educació en la modernitat líquida. Barcelona: Arcàdia.

Blasco, J. y Casado, D. (2011). Avaluació dels Plans Educatius d'Entorn. Ivàlua: Institut Català d'Avaluació de Polítiques Públiques. Recuperado de: http://www.ivalua.cat/ documents/1/17_05_2011_10_04_09_ informe_PEE_def.pdf

Bosch, C., Bravo, F., Alonso, J., Bertran, R., Nieto, J. y Bustos, I. (2008). El trabajo de promoción de redes municipales desarrollado por la Diputación de Barcelona. Cultura y Educación, 20(3), 279-290.

Castells, M. (2004). The Network Society: A Cross-cultural Perspective. Londres: Edward Elgar.

Civís, M., Longás, E., Longás, J. y Riera, J. (2007). Educación, territorio y desarrollo comunitario. Prácticas emergentes. Revista Educación Social, 36, 13-24.

Collet, J. (2009). El treball socioeducatiu en xarxa: una breu proposta de marc conceptual. Congrés de Formació per al Treball en Xarxa a la Universitat. Recuperado de: http://www.fbofill.cat/ trama/pdfs/congres/marc_conceptual_ treball_xarxa-collet.pdf.

Díaz-Gibson, J. y Civís, M. (2011). Redes Socioeducativas promotoras de capi- tal social en la comunidad: un marco teórico de referencia. Cultura y Educación, 23(3), 415-429.

Díaz-Gibson, J., Civís, M., Longás, J. y Murat, A. (2010). The Study of Educative Network organization in the City of Barcelona, Spain. The Nou Barris District. International Journal of Knowledge Society Research. 1(2), 26-37.

Díaz-Gibson, J., Civís, M. y Longás, J. (2012). La Gobernanza de Redes Socioeducativas: claves para una gestión exitosa. Manuscrito no publicado en proceso de revisión.

Edelenbos, J. y Klijn, E. (2007). Trust in complex decision-making networks: A theoretical and empirical explanation. Administration and Society, 39, 25-50.

Eggers, B. y Singh, S. (2009). The Public Innovators Playbook: Nurturing Bold Ideas in Government. Washington, DC, EE. UU: Ash Institute.

Fernández Enguita, M. (2011). L'educació en temps de crisi. Barcelona: Centre d'Estudis Jordi Pujol.

Fundació Pi i Sunyer (2011). Banc de Bones Pràctiques. Recuperado de: http://www.bbp.cat.

Generalitat de Catañunya (2012). Els Plans Educatius d'Entorn. Recuperado de: http://www20.gencat.cat/docs/Educacio/Home/Arees_actuacio/innovacio_educativa/Plans $\% 20$ educatius $\% 20$ entorn/doc_marc_pee_cat.pdf.

Hinterhuber, A. (2002). Value Chain Orchestration in Action and the Case of the Global Agrochemical Industry. Long Range Planning, 35(6), 615-635.

Klijn, E., Edelenbos, J. y Steijn, B. (2010). Trust in Governance Networks: 
Its Impacts on Outcomes. Administration and Society, 42(2), 193-221.

Longás, J. y Civís, M. (coord.) (2009). Xarxes socioeducatives, treball en xarxa $i$ transversalitat. Barcelona: Fundació Blanquerna.

Longás, J. y Civís, M. (coord.) (2010). Estudi de les xarxes socioeducatives de Nou Barris. Fortaleses i oportunitats. Informe de recerca. Barcelona: Universitat Ramon Llull.

Longàs, J., Civís, M. y Riera, J. (2008). Asesoramiento y desarrollo de redes socioeducativas locales. Funciones y metodología. Cultura y Educación, 20(3), 303-324.

Longás, J., Fontanet, A. y Bosch, M. (2007). La organización en red como respuesta a las necesidades socioeducativas de una comunidad. El caso de Sant Vicenç dels Horts. Educación Social, 36, 52-69.

Longo, F. (2008). Liderazgo distribuido, un elemento crítico para promover la innovación. Capital humano: revista para la integración y desarrollo de los recursos humanos, 226, 84-91.

Miller, P., Díaz-Gibson, J., Miller-Baslev, G. y Scalan, M. (2012). Looking beyond Harlem: International insights for area-based initiatives. Middle School Journal, September, 16-24.

Miller, G. y Jæger, B. (2011). Organizational innovation in the creation of new Methods for retaining young people in education. Innovation in public service - a nordic cooperation-. Lillehammer Folkhighschool, Karlstad University, Roskilde University.

Milward, H. B. y Provan, K. G. (2006). A manager's guide to choosing and using collaborative networks. IBM Endowment for the Business of Government Recuperado de: http://www.busines- sofgovernment.org/sites/default/files/ CollaborativeNetworks.pdf.

Morin, E. (2004). Introducción al pensamiento complejo. Barcelona: Gedisa.

Renée, M. y Mcalister, S. (2011). The Strengths and Challenges of Community Organising as an Education Reform Estrategy: What the Research Says. Quincy: Nellie Mae Education Foundation.

Saz, A. (2007). Managing Interorganizational Networks: Leadership, Paradox and Power. Cases from the U. S. Inmigration Sector. Tesis Doctoral. Barcelona: Universitat Ramon Llull.

Sorensen, E. y Torfing, J. (2009). Making governance networks effective and democratic through metagovernance. Public Administration, 87(2), 234-258.

Sorensen, E. y Torfing, J. (2011). Enhancing Collaborative Innovation in the Public Sector: An Analytical Framework. Administration and Society, 43(8), 842-868.

Subirats, J. y Albaigés, B. (coord.) (2006). Educació $i$ comunitat. Reflexions a l'entorn del treball integrat dels agents educatius. Barcelona: Fundació Jaume Bofill.

Ubieto, J. R. (2007). Modelos de trabajo en red. Educación Social, 36, 26-39.

Ubieto, J. R. (2009). El Trabajo en Red. Usos posibles en Eduación, Salud Mental y Atención social. Barcelona: Gedisa.

Ubieto, J. (2012). La construcción del caso en el Trabajo en Red. Teoría y práctica. Barcelona: Editorial UOC.

Vilar, J. (2008). Implicaciones éticas del trabajo en red y la acción comunitaria. Cultura y Educación, 20(3), 267-277.

Waissbluth, M. (2008). Sistemas complejos y gestión pública. Recuperado de: http://www.mariowaissbluth.com/descargas/complejidad_y_gestion.pdf. 


\section{PERFIL ACADÉMICO Y PROFESIONAL DE LOS AUTORES}

Mireia Civís Zaragoza, Maestra, pedagoga y doctora en pedagogía, profesora titular de la Facultad de Psicología, Ciencias de la Educación y del Deporte Blanquerna (Universidad Ramon Llull). Con experiencia tanto en el ámbito de la pedagogía social como de la pedagogía escolar. Miembro del Grupo Consolidado de Investigación PSITIC de la Universidad Ramon Llull. Sus líneas prioritarias de investigación se centran en torno al desarrollo de redes socioeducativas y de proyectos educativos comunitarios, siendo también núcleos de interés la corresponsabilidad educativa y la participación ciudadana. Ha escrito varios artículos y libros vinculados a estas temáticas así como ha llevado a cabo proyectos de investigación y participado en asesoramientos diversos.

Jordi Longás Mayayo, educador social, pedagogo y doctor en pedagogía, Profesor de la Facultad de Psicología, Ciencias de la Educación y del Deporte Blanquerna (Universidad Ramon Llull). Coordinador académico del Máster Universitario de Pedagogía Social y Comunitaria: Liderazgo de la Transformación Socioeducativa. Miembro del Grupo Consolidado de Investigación PSITIC de la Universidad Ramon Llull. Asesor de la Diputación de Barcelona para la creación de Redes Educativas Locales y mejora del éxito escolar en diversos municipios de Cataluña. Sus líneas prioritarias de investigación se vinculan a la organización escolar y bienestar docente, y al desarrollo de redes educativas y desarrollo comunitario. Ha publicado varios artículos y libros vinculados a estas temáticas.

Dirección de los autores: Mireia Civís Zaragoza

FPCEE Blanquerna, Universitat Ramon Llull

C/ Císter, 34

08022 Barcelona

e-mail: Mireiacz@blanquerna.url.edu

JordiLM@blanquerna.url.edu

Fecha Recepción del Artículo: 1. Junio. 2012

Fecha Modificación Artículo: 18. Diciembre. 2012

Fecha Aceptación del Artículo: 22. Enero. 2013

Fecha Revisión para publicación: 15. Mayo. 2014 
